\title{
Editorial
}

\section{La enseñanza de la economía en las disciplinas sociales}

No se evidencia hoy un circuito dialéctico entre lo que la disciplina económica tiene de las ciencias sociales y lo que esta aporta a las mismas. Los currículos de las disciplinas sociales transitan en sendas hiper-especializadas que no dialogan entre sí. No se identifica el aporte que unas y otras se hacen, llevando a que su enseñanza sea desconectada, y que por lo tanto, los profesionales de unas y otras, cuando emprenden trabajos interdisciplinarios tengan profundas complicaciones para construir un discurso común. En el mismo sentido, el carácter moral de la economía se ha opacado por la insistencia en el uso de instrumental matemático y estadístico de alto nivel, lo cual deja en algunas ocasiones por fuera las discusiones fundamentales. Podríamos, de manera muy superficial indicar que existen cuatro grandes discusiones que pueden llevar a una mejor comprensión de lo que necesitamos de la enseñanza de la economía en las ciencias sociales, y lo que estas pueden dar para construir un puente interpretativo útil para las nuevas generaciones.

Discusión No.1: ¿Se requiere elementos de la disciplina económica para la vida profesional de los ciencistas sociales? Los ciencistas sociales (historiadores, antropólogos, sociólogos, contables, administradores, trabajadores sociales, entre otros) requieren de elementos propios de la disciplina económica para su propia formación, ese es un aspecto ya superado, el problema es: ¿Qué tipo de conocimientos necesitan? En los currículos observamos dos tipos de tendencias, una primera en la que se incluyen elementos básicos de economía política, incluso de política económica como aspectos generales de contextualización sin una conexión adecuada con los instrumentales que los profesionales de la economía están utilizando en la actualidad. La otra tendencia es la inclusión de elementos mínimos de la micro y macro economía sin conexión con la política económica, y peor aún, con las implicaciones que esto tiene para las otras disciplinas. Tanto una postura como la otra, han creado una dificultad importante en otros profesionales no economistas al no permitirles dimensionar realmente la importancia y funcionalidad, tanto del conocimiento, como del pensamiento económico contemporáneo.

Como resultado de esto, el instrumental que los economistas han desarrollado en las últimas décadas para la interpretación y gestión, sobre todo, de las políticas públicas resulta ajeno, y en algunos casos incomprensible. Existe aquí también una seria responsabilidad de algunos de los educadores que portan el saber económico, al asumir su conocimiento como algo fijo, inmutable al que los demás deben acceder, y no como algo que es maleable y que sirve para construir puentes con otros saberes. 
Discusión No. 2: ¿Dónde se genera epistemológicamente conocimiento económico y cómo puede hoy ser reconocido por portadores de otras disciplinas y profesiones? Quienes enseñan la disciplina económica hoy conviven en dos mundos altamente complejos. Un primer mundo que valora ampliamente el conocimiento epistemológico, el cual tiene que ver con la enseñanza parcial de la economía clásica y hasta de los fundamentos de la economía neoclásica. Por otro lado, coincide un segundo mundo, dominado por los constructores de instrumentales derivados de la economía neoclásica, que alimentan hoy por hoy, un importante número de journals internacionales. Publicaciones donde las autoridades académicas se legitiman.

Pareciera entonces que los profesionales en economía han decidido vivir en uno de estos dos mundos, y mientras los más experimentados siguen defendiendo el primer mundo epistémico, los más jóvenes acosados por el academicismo defienden a capa y espada el segundo. A la larga hoy no se es nadie por lo que se sepa, sino por lo que se publica. Esto ha creado un muro infranqueable para otras disciplinas, en el cual es muy difícil, incluso imposible para algunos, entrar en discusión con lo que se escribe en los journals contemporáneos. El eco se da en aquellos pocos que trabajan la primera postura.

Hoy los economistas posan como doctos en miles de áreas (educación, salud, relaciones humanas, etc.), pero son incapaces de establecer un dialogo con los sujetos que alimentan discusiones y enfrentan problemáticas desde ámbitos profesionales ajenos. Así la forma como se produce conocimiento económico está cerrado a un circuito académico delimitado, que deja por fuera a propios y extraños, haciendo que su aislamiento se agudice, sin pensar que su capacidad de innovación radical en conceptos esté en duda. Esto queda de manifiesto, porque se produce mucho conocimiento, pero con un valor interpretativo muy limitado. Se termina dando mucho valor a la técnica.

Discusión No. 3: ¿Cuáles son los límites del pensamiento económico contemporáneo y dónde otras disciplinas hoy muestran una mejor eficacia para comprender la realidad? La enseñanza de la economía hoy debería ser muy clara en establecer cuáles son sus límites epistémicos, conceptuales e instrumentales. Economistas y profesionales de otras disciplinas tendrían que mediar en mirar esos límites y en construir puentes de trabajo conjunto. Tal proceso no se está haciendo, y como tal, quienes se forman en ciencias económicas tampoco reciben los insumos suficientes para tener una comprensión de la realidad más compleja y rica, lo que interrumpe el dialogo de saberes.

La inclusión de manera autónoma de saberes como la estadística en áreas como la antropología, la sociología y la contabilidad, las escuelas criticas de pensamiento administrativo, las nuevas líneas de estudios del trabajo, y la misma necesidad de construir identidades y subjetividades pueden aportar elementos valiosos a la enseñanza de la economía, que como tal, le ayudarían a integrar elementos que van más allá de la construcción y testeo de los modelos, es decir, más allá de la técnica.

Muchas de las disciplinas sociales contemporáneas han logrado niveles de intervención de la realidad social, que a nivel micro resultan muy eficaces, por ejemplo en temas como los presupuestos participativos, los procesos de accontability para la gestión social y el seguimiento de normativas voluntarias para la mejora de gestión ambiental en organizaciones. Aspectos ajenos a la formación del economista, creándole una imagen de técnico, que 
tiene la posibilidad de establecer políticas basadas en información institucional sin tener en cuenta las comunidades, o en el peor de los casos con un profundo desconocimiento del terreno, en otras palabras, que corre modelos pero de espaldas a la realidad social.

Discusión No. 4: Se requiere de una enseñanza de la economía que tenga diálogo con otros saberes tanto para sus iniciados, como para los profanos de origen. En realidad este último punto es el resultado de los tres anteriores. El papel de los formadores, de alguna manera invocado anteriormente, es importante, al mismo tiempo que el de los planificadores educativos, quienes en función de la eficiencia han sacrificado aspectos que podrían resultar importantes en la formación de los economistas (ej.: filosofía, historia, ciencia política, contabilidad, etc.) y de otros profesionales en ciencias sociales. Se ha apostado por un saber disciplinar propio y depurado que privilegia las micros, macros, econometrías, economías matemáticas, etc.

Las reformas educativas contemporáneas han ido en esta dirección, dándose una reducción de los contenidos observados, una disminución de los tiempos de formación y el hecho de dar un valor desmesurado a la escritura formal para revistas científicas seriadas. Como resultado, tenemos estudiantes autómatas, muy eficientes en la escritura, pero con pocas experiencias de realidad aportadas por la universidad. Es fundamental integrar además de la formación socio-humanista (para la tan cacareada formación integral) una serie de actividades que permitan al estudiante interactuar con otros escenarios sociales. La enseñanza de la economía requiere de pasantías profesionales y/o sociales, donde se reconozcan las exigencias del medio. En complemento, debería ofrecerse la posibilidad de adquirir alguna formación artística básica, que permita establecer ámbitos de sensibilidad, útiles y necesarios para la construcción de políticas públicas que escapan de la racionalidad y que van hacia la razonabilidad.

En síntesis, la enseñanza de la economía requiere del trabajo en equipo para la solución de problemas concretos, siendo relevante por ejemplo el aprendizaje basado en proyectos, sobre todo ahora, cuando en el discurso oficial apremian las habilidades en creatividad para la innovación y la perseverancia para el emprendimiento. La enseñanza de la economía no se puede quedar únicamente en la formación de técnicos para el aparato burocrático del Estado, habiendo sido esa la necesidad básica para la expansión de escuelas hace algunas décadas, ni en la formación de matemáticos y estadísticos por adopción. En cambio debe promover ciudadanos comprometidos en la discusión y construcción de soluciones y alternativas a las diversas problemáticas, con dinámicas de trabajo que redunden en un reconocimiento del conocimiento económico en las comunidades de base. Es el desarrollo de tales competencias, en muchos sentidos, la tarea que docentes, investigadores y administradores educativos de las ciencias económicas deberían plantearse.

Edison Fredy León Paime Editor

David Andrés Camargo

Co-editor 